\title{
Extending the Detection Limit for Near Infrared Spectroscopic Measurements of Heterogeneous Solids Using Focal Plane Array Detectors
}

\author{
E. Neil Lewis ${ }^{*}$, Eunah Lee ${ }^{*}$, Linda H. Kidder ${ }^{*}$, Everett H. Jefferson ${ }^{* *}$, Christopher D. Ellison ${ }^{* *}$, \\ Patrick J. Faustino $^{* *}$, Robbe C. Lyon ${ }^{* *}$, and Ajaz S. Hussain ${ }^{* * *}$ \\ *Spectral Dimensions, Inc., 3416 Olandwood Court, Suite 210, Olney, MD 20832 \\ *** Division of Product Quality Research, Food and Drug Administration, Kensington, MD 20895 \\ ***Office of Pharmaceutical Sciences, Food and Drug Administration, Rockville, MD 20857
}

NIR chemical imaging instrumentation has the potential to detect and identify trace constituents in heterogeneous samples more quickly and at lower limits of detection than attainable using conventional near-infrared spectroscopic systems. Instead of a single point detector, an NIR imaging system uses an infrared focal-plane array (FPA) detector to rapidly generate up to 81,900 spatially resolved spectra in a single data set. The resulting images and pixel spectra allow visualization of the distribution of chemical constituents comprising the sample.

Conventional NIR spectroscopic analysis of a sample with a contaminant results in a single 'average' spectrum representing the entire object, which is a mixture spectrum of the matrix and the contaminant. Under these bulk measurement conditions, the ability to find the contaminant represents a dilution problem. As the size of the contaminant relative to the substrate decreases, the contribution to the total spectrum decreases, and the detection limit is rapidly reached, even for high signal to noise ratio data. A partial solution to this problem lies in the use of near-infrared microanalysis, but the entire sample has to be mapped sequentially. This approach, while extremely sensitive, requires long data collection times and is usually considered prohibitive for routine analysis. NIR spectral imaging using a focal-plane array solves the problem by sampling all spatial locations simultaneously. There is no time penalty for analyzing the entire sample and there is no 'dilution' since a separate spectrum is recorded for each spatial location.

An NIR imaging data set of a single pharmaceutical tablet consisting of a mixture of a drug (furosemide, 20\%) and a standard excipient (Avicel 80\%) was collected in reflectance mode and divided by a 99\% reflectance background data set (Spectralon, Lab Sphere, North Sutton, NH) to generate reflectance image cubes. The resulting reflectance image cube is further transformed into absorbance data using $\log (1 / \mathrm{R})$. The data were collected using a Matrix NIR imaging system (Spectral Dimensions, Olney, MD) over the spectral range 1150-1700 nm with $7 \mathrm{~nm}$ increments. Each data set containing 76,800 individual spectra takes approximately 2 minutes to collect. A single pixel spectrum corresponds to an area of approximately 40x40 microns on the sample surface. Figure 1 shows pure component near-infrared spectra collected of the two major components (red and blue traces), and the spectrum of furosemide related compound B (USP) (dotted green trace), a potential impurity that results from the chemical degradation of the drug. Figure 2a is a chemical image that shows the distribution of the impurity in the tablet. The image contrast is based on the strength of the normalized absorption band at $1440 \mathrm{~nm}$, a spectral feature unique to the impurity. Figure $2 \mathrm{~b}$ shows a single pixel spectrum from within the tablet after subtraction of a normalized matrix spectrum, and clearly identifies the bright regions in the image as those associated with the contaminant. From a simple pixel abundance calculation we can assess the amount of the impurity on the surface of the tablet at approximately $0.5 \%$. This is a concentration level that would be 
challenging to detect using a conventional bulk near-infrared spectroscopic method. For the data presented, the spectrum of the contaminant was evident on 216/40484 pixels. Extrapolating to a detector with $>300,000$ detectors, as long as a single pixel containing an anomalous spectrum could be identified, the detection limit could easily be $3 \mathrm{ppm}$ without the need for high signal to noise spectral data. As the number of pixels increases the total amount of sample that is screened in a single measurement increases and therefore the relative 'concentration' that is detected decreases. This suggests that, for an NIR imaging experiment, the detection limit is largely determined by the number of detectors (pixels) employed. Further, if there are specific absorption bands unique to the impurity, a complete spectrum at each pixel location does not need to be collected. Data collected at a few 'marker' wavelengths can provide the 'answer' in a matter of a few seconds. Further details of these novel approaches for quantitative and 'trace' NIR analysis will be presented.

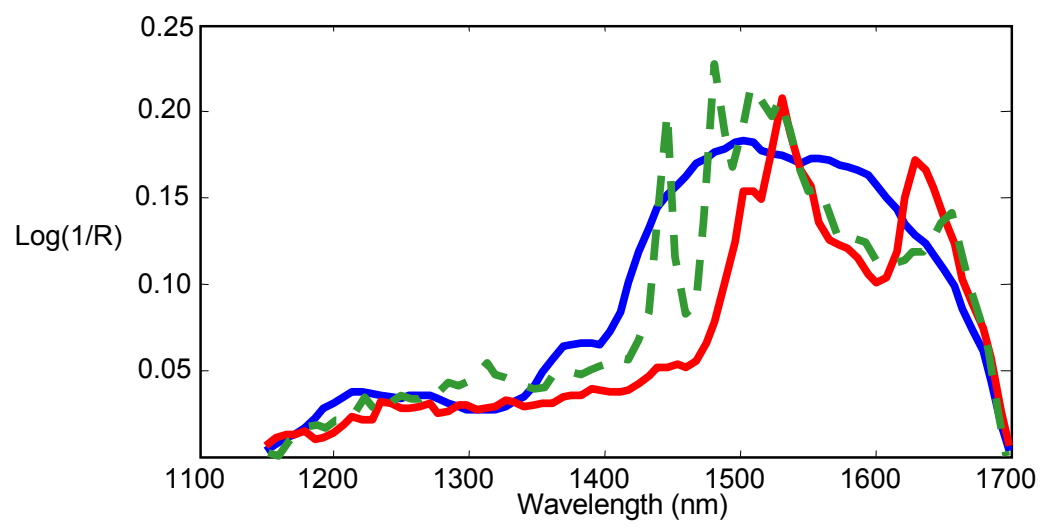

Figure 1: Pure component spectra of the tablet constituents. The red trace is the spectrum of Furosemide, the active pharmaceutical ingredient. The blue trace is the spectrum of Avicel, a standard excipient. The green dotted trace is the spectrum of furosemide related compound B.
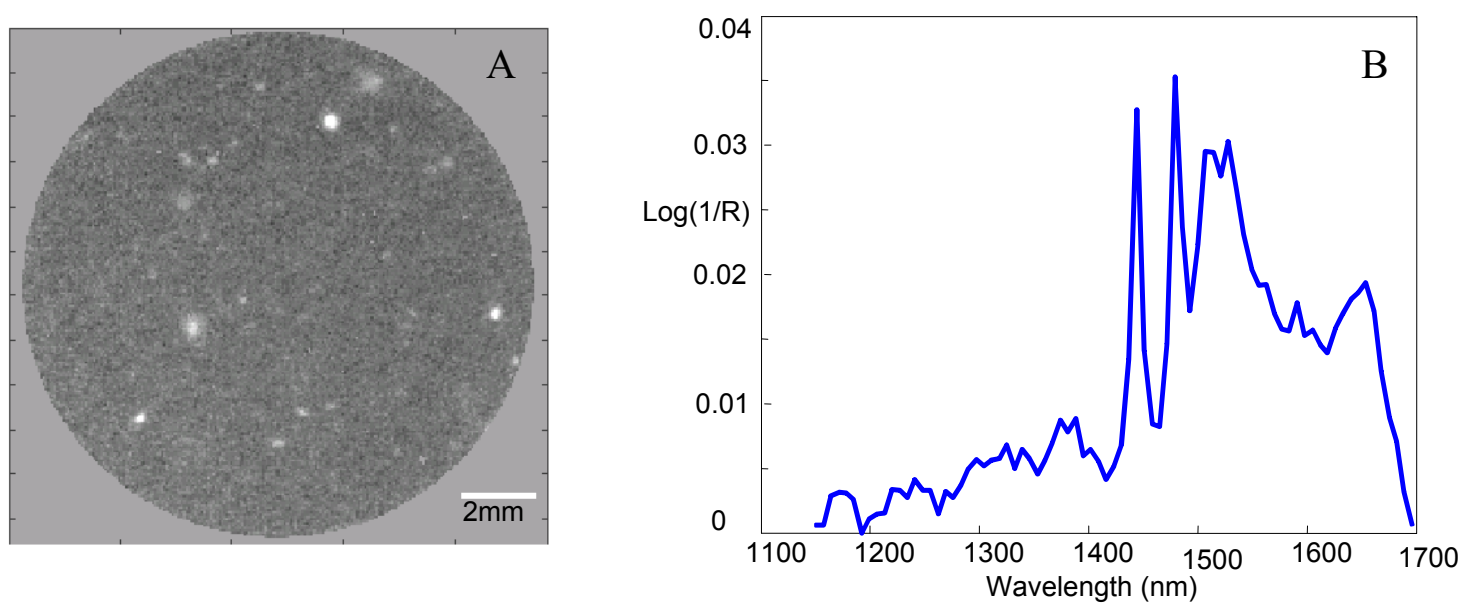

Figure 2. Figure 2a is a chemical image of the whole tablet at $1440 \mathrm{~nm}$. The image contrast is based on the relative strength of sample absorption at this wavelength relative to that at $1470 \mathrm{~nm}$. As the impurity has a strong, unique spectral feature at $1440 \mathrm{~nm}$, these particles appear bright. Figure $2 \mathrm{~b}$ is the spectrum from a single pixel within one the bright spots after subtraction of a normalized matrix spectrum. The impurity is estimated to be present at $216 / 40484$ pixels $(0.5 \%$ abundance $)$. 